\title{
Hemispheric processing of inferences: The effects of textual constraint and working memory capacity
}

\author{
SANDRA VIRTUE \\ DePaul University, Chicago, Illinois \\ PAUL VAN DEN BROEK \\ University of Minnesota, Minneapolis, Minnesota \\ and \\ TRACY LINDERHOLM \\ University of Florida, Gainesville, Florida
}

\begin{abstract}
In this study, we investigated hemispheric differences in the generation of bridging and predictive inferences. Participants read texts that provided either strong or weak causal constraints for a particular bridging (Experiment 1) or predictive (Experiment 2) inference and performed a lexical decision task to inference-related targets presented to the left or the right hemisphere. Facilitation for strongly constrained bridging and predictive inferences was found in both hemispheres. In contrast, facilitation for weakly constrained inferences was stronger in the right than in the left hemisphere for both bridging and predictive inferences, although for the latter there was some facilitation in the left hemisphere as well. We also considered whether these effects differ as a function of the working memory capacity of the reader. High working memory capacity readers showed greater facilitation for strongly constrained inferences than for weakly constrained inferences in both hemispheres, whereas low working memory capacity readers showed this same pattern in the left hemisphere but showed equal facilitation for strongly and weakly constrained inferences in the right hemisphere. These results suggest that hemispheric processing, textual constraint, and working memory capacity interact to affect how readers generate causal inferences.
\end{abstract}

Much of our comprehension of text is derived from the making of inferences. To make inferences, we must connect explicit text events with other text events or with our background knowledge. The result of this process is the formation of a coherent mental representation. One important factor influencing inference generation is the causal structure of text events (see, e.g., Graesser, Singer, \& Trabasso, 1994; van den Broek, 1994). Little is known, however, about how the cerebral hemispheres respond to causal information when generating inferences to form coherence. In this study, we investigated whether the left and right

This research was supported by the Center for Cognitive Sciences at the University of Minnesota through Grant HD-07151 from the National Institute of Child Health and Human Development, by the Guy Bond Endowment for Reading and Literacy, and by a Golestan fellowship at the Netherlands Institute for Advanced Study in the Humanities and Social Sciences to P.v.d.B. We thank Chad Marsolek and Brian Sundermeier for their helpful suggestions throughout the course of this research. We also thank Pha Lee and Elizabeth Couchet for their assistance in collecting data. Correspondence concerning this article should be addressed to S. Virtue, Department of Psychology, DePaul University, 2219 North Kenmore Ave., Chicago, IL 60614 (e-mail: svirtue@depaul.edu).

Note-This article was accepted by the previous editorial team, when Colin M. MacLeod was Editor. hemispheres play unique roles in causal inference generation. Specifically, we examined two factors that could affect inference generation in the hemispheres: whether an inference is bridging or predictive and whether the inference is strongly or weakly constrained by the text's causal structure. We also explored how the effects of these two factors might differ as a function of the working memory capacity of the reader, an individual-differences component known to affect the causal inferential process.

Although the left hemisphere is considered the dominant hemisphere in the processing of language, the right hemisphere is involved in processes that could contribute to the generation of inferences. For example, righthemisphere-damaged patients have difficulty answering true/false questions about inferable information, despite being able to answer questions about explicitly stated information (Brownell, Potter, Bihrle, \& Gardner, 1986). Furthermore, unlike non-brain-damaged readers, they show longer response times to inference-related words than to unrelated words after reading stories that promote inferences (Beeman, 1993). In addition, the right hemisphere is highly activated when participants comprehend metaphors versus literal sentences (Bottini et al., 1994), read untitled texts versus titled texts (St. George, Kutas, Martinez, \& Sereno, 1999), and process connected dis- 
course versus unrelated discourse (Robertson et al., 2000). Thus, the right hemisphere may be important in helping readers maintain coherence while they comprehend texts.

The idea that the right hemisphere is involved in maintaining coherence leads to interesting hypotheses about how the hemispheres process inferences. It is possible that the two hemispheres differ in the activation of semantic information during inference generation. One generally accepted method for studying the activation of semantic information in the hemispheres is the divided visual field paradigm. The divided visual field paradigm is useful because it allows researchers to present material in isolation to one visual field, which leads to processing in the contralateral hemisphere (Church \& Chiarello, 1988; Iacoboni \& Zaidel, 1996). This paradigm has been used to investigate the hemispheric activation of semantic information. For example, when participants are presented with strongly associated words (e.g., scissors, followed by $c u t$ ), target words (cut in the example) show activation in the left and the right hemispheres. In contrast, when participants are presented with weakly associated words (e.g., cry, foot, and glass, followed by cut), target words (e.g., cut in the example) show greater activation in the right hemisphere than in the left hemisphere (Beeman et al., 1994). On the basis of results like these, it has been proposed that the right hemisphere specializes in coarse semantic coding, whereas the left hemisphere specializes in fine semantic coding. In this view, the left hemisphere activates only a few semantic associates of a word, whereas the right hemisphere activates many related semantic associates of a word. During inference generation, readers typically bridge gaps in a text by making connections between distantly related concepts. Therefore, this view supports several predictions about how inference generation occurs in the two hemispheres. The left hemisphere would be less likely to be involved in inference generation because the left hemisphere activates more closely related concepts, which leads to little semantic overlap between concepts. The right hemisphere would be more likely to be involved in inference generation because the right hemisphere broadly activates concepts, which leads to greater semantic overlap between concepts than occurred in the left hemisphere. Presumably, the greater semantic overlap in the right hemisphere enables it to more extensively generate inferences.

Readers engage in the generation of different types of inferences during text comprehension (for taxonomies, see Graesser et al., 1994; Singer, 1990). Two types of inferences are particularly central to the coherence of a text: bridging inferences (or backward inferences) and predictive inferences (or forward inferences; see, e.g., Graesser \& Clark, 1985; van den Broek, 1990). Bridging inferences allow readers to establish explanatory connections between a focal event and prior text or background knowledge. For example, a bridging inference must be generated to comprehend the following sentences: "The man threw the vase against the wall. It cost over one hundred dollars to replace." To understand the second sentence, readers must refer to the first sentence to understand that the vase broke. Readers spontaneously generate bridging inferences while comprehending text (e.g., McKoon \& Ratcliff, 1992; O’Brien, Shank, Myers, \& Rayner, 1988; Singer \& Ferreira, 1983) and incorporate information related to bridging inferences into their mental representation of a text (Johnson, Bransford, \& Solomon, 1973).

In contrast to bridging inferences, predictive inferences allow readers to generate expectations about what will happen next in the text. To anticipate upcoming events in a text, readers must connect information from the text with their background knowledge. For example, when readers encounter the sentence, "The minister just pronounced the couple as husband and wife," they are likely to retrieve information about weddings from their background knowledge to infer that the couple will kiss next. Predictive inferences may be useful for building situation models of text events because, by definition, these inferences require that readers connect text events with background knowledge, which is a key step in forming situation models (Fincher-Kiefer, 1995).

Given recent observations about hemispheric specialization in the processing of semantic information, one might expect the hemispheres to be differentially involved in the generation of bridging and predictive inferences. For example, when participants listened to stories and named aloud target words related to an inference, unique activation was found in each hemisphere (Beeman, Bowden, \& Gernsbacher, 2000). Greater levels of activation for bridging inferences were found in the left hemisphere, whereas greater levels of activation for predictive inferences were found in the right hemisphere.

It is possible that the observed difference between bridging and predictive inference generation in the hemispheres is influenced by the level of causal constraint present in a text, in addition to the type of inference. Causal constraint refers to how strongly text events point to a particular word or event. Indeed, there is evidence that highly constrained information differentially influences how the hemispheres process text. For example, the left hemisphere shows different activation patterns for sentences that have a high or low probability of ending with a certain word, whereas the right hemisphere shows similar activation patterns under both of these conditions (Faust \& Kravetz, 1998). Thus, the left hemisphere is highly activated when participants are presented with linguistically constrained sentences, such as "The cop caught the thief," whereas the left hemisphere is less activated when participants are presented with less constrained sentences, such as "The car was opened by the thief." Also, the left hemisphere shows higher activation for well-structured sentences than for scrambled sentences, whereas the right hemisphere shows similar activation in both conditions (Faust, Babkoff, \& Kravetz, 1995). In summary, the right hemisphere seems capable of processing both weakly and strongly constrained semantic information, whereas the left hemisphere may be more specialized for processing strongly constrained semantic information. 
There is considerable behavioral evidence that textual constraint influences bridging and predictive inference generation. For example, participants showed increased recall for bridging inference sentences that were moderate in causal relatedness than for sentences that were low in causal relatedness (Myers, Shinjo, \& Duffy, 1987). In addition, when participants were presented with strong, moderate, and weak causal constraint predictive inference items, they responded more quickly to inference-related targets after reading strongly constrained text, in contrast to weakly or moderately constrained text (Linderholm, 2002; van den Broek \& Huang, 1995). Thus, bridging and predictive inferences are more likely to be generated when causal constraints are strong (see the causal inference maker model; van den Broek, 1990). Therefore, the level of causal constraint in a text is likely to influence the production of bridging and predictive inferences in the hemispheres.

The effects of textual constraint on hemispheric processing may be influenced by individual differences in working memory capacity. There is considerable evidence that low and high working memory capacity readers differ in their processing of causal inferences (Linderholm, 2002; Linderholm \& van den Broek, 2002; Monzó \& Calvo, 2002; Singer, Andrusiak, Reisdorf, \& Black, 1992; St. George, Mannes, \& Hoffman, 1997). Moreover, although a variety of reader factors influence how individuals generate inferences (e.g., a reader's background knowledge, goal for reading, etc.), a reader's working memory capacity has explicitly been shown to influence how inferences are generated under varying levels of textual constraint. Specifically, the constraint of a text functions differently for readers with different working memory capacities (Linderholm, 2002). Given that the effects of constraint interact with the working memory capacity of individual readers, we explored in a secondary analysis the possibility that observed hemispheric differences in the processing of weakly and strongly constrained causal inferences may vary as a function of working memory capacity.

In the experiments presented in this study, we expected the right visual field-left hemisphere (rvf-LH) to have an overall advantage in response time in the processing of bridging and predictive inferences because the left hemisphere is dominant for language tasks (e.g., Chiarello, Senehi, \& Soulier, 1986; Faust, Kravetz, \& Babkoff, 1993). Therefore, the dependent measure in Experiments 1 and 2 was the inference-related facilitation effect in each visual field-hemisphere. A neutral condition, which shows activation for target words under a noninference condition, was created to provide a baseline of activation for each visual field-hemisphere. The inference-related facilitation effect was obtained by calculating the difference between the neutral and the inference condition response times in each visual field-hemisphere. Only after calculating these inference-related facilitation effects could language differences be directly compared between the left visual field-right hemisphere (lvf-RH) and the rvf-LH. The use of facilitation effects is a commonly accepted method for assessing language effects in the hemispheres (e.g., Beeman et al., 2000; Coney, 2002).

To summarize, we expected that textual constraint would affect the hemispheric processing of inferences. Both bridging and predictive inferences assist in the creation of a coherent mental representation of a text, and both are influenced by the constraint in a text. Therefore, we hypothesized that bridging and predictive inferences would show similar patterns of inference-related facilitation across the left and right hemispheres and that the different patterns of inference-related facilitation would be due mostly to level of constraint. Specifically, the rvf-LH would show high levels of inference-related facilitation for strongly constrained bridging and predictive inferences, whereas it would show low levels of inference-related facilitation for weakly constrained bridging and predictive inferences. More importantly, we hypothesized that the lvf-RH would show high levels of inference-related facilitation effects for both strong and weak causally constrained bridging and predictive inferences. Furthermore, we considered whether the effects of textual constraint on hemispheric processing of inferences would differ across readers who varied in working memory capacity. In Experiment 1, we explored the influence of textual constraint and working memory capacity on the hemispheric processing of bridging inferences; in Experiment 2, predictive inferences were investigated.

\section{EXPERIMENT 1}

\section{Method}

\section{Participants}

Seventy-two undergraduate students at the University of Minnesota participated in the experiment in exchange for course credit in an introductory psychology course. Of these, 36 were male and 36 were female (17-26 years of age). All the participants had normal or corrected-to-normal vision and were native speakers of American English. All were right-handed, as assessed through the Edinburgh Handedness Inventory (mean laterality quotient $=.82$; Oldfield, 1971).

\section{Materials}

Texts. The materials consisted of three sets of 48 texts (inference, neutral, and filler), some adapted from prior research (Cook, Limber, \& O'Brien, 2001; van den Broek \& Huang, 1995) and others created specifically for this study. Each of the 144 texts consisted of four sentences. The last sentence in the inference texts invited a bridging inference. These texts came in strong and weak causal constraint versions. We verified the causal constraint manipulation in a pilot study by asking 33 naive readers what caused the last event in each text. In this pilot study, responses were rated with a 3 if the response included the target inference word, a 2 if the response included a synonym of the inference word, a 1 if the response implied that the inference occurred, and a 0 if the response did not include the target inference word. The average number of target inference words was then calculated for each constraint version. The results confirmed that the target word was more often mentioned as part of the causal explanation for the strong constraint version $(M=9.66$, $S E=0.59)$ than for the weak constraint version $(M=1.53, S E=$ $0.35)[t(47)=15.24, S E=0.53, p<.01]$. Importantly, the average responses to the strong and weak constraint texts were significantly greater than would have been expected if the readers had been gener- 
ating inferences by chance. In addition, the average responses to the strong and weak constraint texts were significantly greater than zero $[t(47)=16.40, S E=0.59, p<.05 ; t(47)=4.39, S E=0.35, p<$ $.05]$. The neutral and filler texts covered miscellaneous topics that did not promote these bridging inferences. Examples of an inference text in its strong and weak constraint versions and of a neutral text are given in Table 1.

Target words. Each of the inference and neutral texts had a corresponding target word that represented the targeted inference (e.g., spray). The neutral texts were paired with target words that represented the inference, but these neutral texts did not promote the targeted inference. For example, for the neutral text in Table 1, it is unlikely that readers would generate the targeted bridging inference spray after reading "They often rode around town in the shiny red Mustang." Therefore, the neutral texts provided a baseline of performance in each hemisphere. The target words were one- or twosyllable action verbs and were similar in number of letters and word frequency across the texts (based on Francis \& Kučera, 1982). The filler texts did not promote inferences and were paired with nonword targets. Data for the filler texts were excluded from the analyses.

\section{Procedure}

The participants read an equal number of texts from each set (e.g., inference, weakly or strongly constrained; neutral; or filler). In addition, the participants received an equal number of weakly and strongly constrained texts. The order in which the texts were read and the type of textual constraint (weak or strong) were counterbalanced with six between-subjects counterbalancing conditions. The participants read each text on the computer one sentence at a time, in a self-paced manner. The participants were instructed to fixate on the center of a fixation plus $(+)$ for the entire time it appeared on the computer screen $(750 \mathrm{msec})$. This time parameter was chosen because it provided the participants with sufficient time to generate an inference (Till, Mross, \& Kintsch, 1988). The participants then performed a lexical decision task in which a target (word or nonword) was presented to the participants' left or right visual field for $176 \mathrm{msec}$ after they had read each text. The rapid presentation of the target was used so that the participants did not have time to move their eyes to the target, thus ensuring that the target appeared in only one visual field-hemisphere. The distance from the participants' eyes to the targets on the computer screen was $50 \mathrm{~cm}$. Targets appeared approximately $3.5^{\circ}$ of visual angle to the left or the right of fixation. The center of each target was $3.4 \mathrm{~cm}$ from the fixation center, and the inner edge of each word was at least $1.5 \mathrm{~cm}$ from the outer edge of the fixation point.

The participants were instructed to decide as quickly and as accurately as possible whether the target they saw formed a word. To

Table 1

Example Bridging Inference Text Used in Experiment 1

\section{Inference Text}

After the rugby match, Justin's friends teased him for not knowing the rules.

He gathered around his friends and joked about beating them next time. In order to look macho, Justin grabbed a beer from the cooler.

Strong textual constraint: His friends were soon covered in beer. Weak textual constraint: His friends were soon cheering him on. Target word: spray

\section{Neutral Text}

The teenage boys were cruising the streets of their town. One of the boys had a nice new sports car.

The girls in town were impressed by the fast car.

They often rode around town in the shiny red Mustang.

Target word: spray ensure that the participants read for comprehension, they were asked to write down the main ideas for some of the texts. Each participant read 48 inference texts (weakly or strongly constrained), 48 neutral texts, and 48 filler texts (with a corresponding target word or nonword for each text), so all the participants read a total of 144 texts and targets in this experiment.

\section{Reading Span Task}

After the participants had finished reading all the texts, they were administered a reading span task (Singer \& Ritchot, 1996) to distinguish low and high working memory capacity readers. In this task, the participants read a set of unrelated sentences, one sentence at a time, and were to recall the final word of each sentence in the set. The participants were then presented with one of the sentences from the set that they had just finished reading and were to recall two words that were missing from the sentence. The total number of final words recalled in the reading span task was used to distinguish low from high working memory capacity readers (see Linderholm \& van den Broek, 2002, for method). When the upper and lower terciles of the distribution of final words recalled are used, 25 participants were identified as low working memory capacity readers $(M=27.15$, $S D=1.08$ ), and 26 participants were identified as high working memory capacity readers $(M=37.19, S D=2.50)$.

\section{Results}

Lexical decision response time and accuracy for the target words were collected and analyzed. Only correct responses were included in the analyses. To minimize the risk of including outliers, response times that were two standard deviations above and below the mean in each condition were not included in the analyses. In all the analyses reported, $F_{1}$ refers to tests based on participant variability, and $F_{2}$ refers to tests based on item variability. An alpha level of .05 was used to determine the significance for all the analyses.

\section{Inference-Related Response Time Facilitation Effects}

To test the main hypothesis that the level of textual constraint influences the processing of bridging inferences, we conducted analyses on the inference-related facilitation effects. We obtained the inference-related facilitation effects by calculating the difference between the neutral and inference condition response times in each visual field-hemisphere. A repeated measures ANOVA was conducted on mean inference-related facilitation and accuracy facilitation. The independent variables were visual field-hemisphere (left or right) and textual constraint (strong or weak). Sex, response hand, counterbalancing condition, and participant were entered as betweensubjects variables for the by-participants analyses (see Pollatsek \& Well, 1995). There was no effect of sex, response hand, or counterbalancing condition, so these analyses are not reported.

The mean inference-related facilitation for strongly and weakly constrained texts by visual field-hemisphere is presented in Figure 1 (see Table 2 for mean response times in each inference condition). ${ }^{1}$ There was a main effect of visual field-hemisphere by participants $\left[F_{1}(1,64)=4.49, M S_{\mathrm{e}}=\right.$ $77,377.71, p<.05 ; F_{2}(1,47)=1.47, M S_{\mathrm{e}}=43,138.44$, $p=.23]$. Overall, the lvf-RH showed greater inference- 


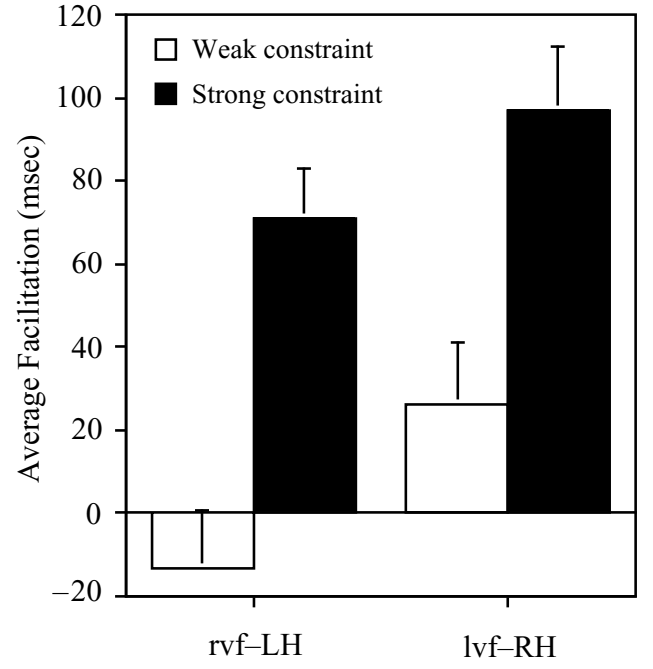

Visual Field-Hemisphere

Figure 1. Average facilitation for strongly and weakly constrained bridging inference texts by visual field-hemisphere (Experiment 1). rvf- $\mathrm{LH}$, right visual field-left hemisphere; Ivf- $\mathrm{RH}$, left visual field-right hemisphere.

related facilitation than did the rvf-LH $(M=61.58, S E=$ 11.24 , and $M=28.82, S E=9.94$, respectively). There also was a main effect of textual constraint $\left[F_{1}(1,64)=60.45\right.$, $M S_{\mathrm{e}}=434,481.98, p<.05 ; F_{2}(1,47)=26.72, M S_{\mathrm{e}}=$ $287,732.04, p<.05]$. There was greater inference-related facilitation for strongly constrained texts than for weakly constrained texts $(M=84.08, S E=9.89$, and $M=6.32$, $S E=10.49$, respectively). There was no visual fieldhemisphere $\times$ textual constraint interaction $\left[F_{1}(1,64)=\right.$ $0.58, M S_{\mathrm{e}}=3,694.36, p=.44 ; F_{2}(1,47)=0.10, M S_{\mathrm{e}}=$ $1,269.63, p=.76]$. However, simple-effect contrasts revealed that weakly constrained inferences showed greater inference-related facilitation in the lvf-RH than in the rvf-LH $\left[F_{1}(1,64)=8.99, M S_{\mathrm{e}}=57,443.46, p<.05\right.$; $\left.F_{2}(1,47)=4.37, M S_{\mathrm{e}}=29,604.69, p<.05\right]$. Thus, both hemispheres showed facilitation of bridging inferences when the text provided strong causal constraints, but the right hemisphere showed greater facilitation of bridging inferences than did the left hemisphere when the text provided weak causal constraints.

One-tailed paired difference $t$ tests were conducted on the bridging-inference-related facilitation effects to determine whether there was facilitation above zero for each level of textual constraint in each visual field-hemisphere. In these analyses, $t_{1}$ refers to tests based on participant variability, and $t_{2}$ refers to tests based on item variability. In the rvf-LH, the inference-related facilitation was significantly greater than zero for strongly constrained texts $\left[t_{1}(71)=5.84, S E=12.18, p<.05 ; t_{2}(47)=3.32\right.$, $S E=22.02, p<.05]$, but not for weakly constrained texts $\left[t_{1}(71)=-0.95, S E=14.11, p=.34 ; t_{2}(47)=-0.43\right.$, $S E=22.45, p=.67]$. In the lvf-RH, the inference- related facilitation was significantly greater than zero for strongly constrained texts $\left[t_{1}(71)=6.26, S E=15.52\right.$, $\left.p<.05 ; t_{2}(47)=4.88, S E=20.04, p<.05\right]$, and the inference-related facilitation in the lvf-RH was marginally significantly greater than zero for weakly constrained texts $\left[t_{1}(71)=1.71, S E=15.26, p=.09 ; t_{2}(47)=1.38\right.$, $S E=18.53, p=.17]$.

\section{Accuracy Response Time Facilitation Effects}

Facilitation of the accuracy responses was inspected to validate the speed-based results (see Table 3 for mean accuracy response times in each inference condition). There was a main effect of visual field-hemisphere $\left[F_{1}(1,64)=\right.$ $8.30, M S_{\mathrm{e}}=0.12, p<.05 ; F_{2}(1,47)=12.19, M S_{\mathrm{e}}=0.08$, $p<.05]$. Overall, accuracy facilitation was greater when target words were presented to the lvf-RH than when target words were presented to the rvf-LH $(M=5.8 \%, S E=$ 0.01 , and $M=1.8 \%, S E=0.01$, respectively). There was no main effect of accuracy facilitation for textual constraint $\left[F_{1}(1,64)=1.04, M S_{\mathrm{e}}=0.01, p=.31 ; F_{2}(1,47)=0.54\right.$, $\left.M S_{\mathrm{e}}=0.01, p=.47\right]$ and no visual field-hemisphere $\times$ textual constraint interaction $\left[F_{1}(1,64)=0.42, M S_{\mathrm{e}}=\right.$ $\left.0.01, p=.52 ; F_{2}(1,47)=0.38, M S_{\mathrm{e}}=0.01, p=.54\right]$. It should be noted that accuracy was very high and close to ceiling. Thus, there was no evidence of a speed-accuracy trade-off.

\section{Working Memory Capacity Effects on \\ Inference-Related Response Time Facilitation}

To explore the effects of working memory capacity, the bridging-inference-related facilitation data were submitted to a $2 \times 2 \times 2$ (working memory capacity $\times$ textual constraint $\times$ visual field-hemisphere) mixed factors ANOVA (see Table 4 for mean response times in each inference condition). Given that working memory analyses were secondary and involved only a subset of the data, items were not balanced across conditions or participants, and therefore, no by-items analyses will be reported. There was a significant three-way interaction of working memory capacity, textual constraint, and visual fieldhemisphere $\left[F(1,51)=6.79, M S_{\mathrm{e}}=5,957, p<.05\right]$.

Table 2

Mean Response Times (in Milliseconds) and Standard Errors for Strong, Weak, and Neutral Textual Constraint Bridging and Predictive Inference Texts by Visual Field-Hemisphere

\begin{tabular}{llllll}
\hline & \multicolumn{2}{c}{ rvf-LH } & & \multicolumn{2}{c}{ lvf-RH } \\
\cline { 2 - 3 } \cline { 6 - 7 } Condition & $M$ & $S E$ & & $M$ & $S E$ \\
\hline Bridging Inference Texts & & & & & \\
$\quad$ Strong textual constraint & 842 & 20.06 & & 845 & 23.31 \\
$\quad$ Weak textual constraint & 926 & 27.75 & & 916 & 23.19 \\
$\quad$ Neutral textual constraint & 913 & 22.12 & & 942 & 23.18 \\
Predictive Inference Texts & & & & \\
$\quad$ Strong textual constraint & 791 & 22.68 & & 843 & 31.38 \\
$\quad$ Weak textual constraint & 854 & 26.19 & & 866 & 25.19 \\
$\quad$ Neutral textual constraint & 883 & 27.52 & & 938 & 32.51 \\
\hline
\end{tabular}

Note-rvf-LH, right visual field-left hemisphere; lvf-RH, left visual field-right hemisphere. 
Table 3

Mean Accuracy Response Times (in Percentages Correct) and Standard Errors for Strong, Weak, and Neutral Textual Constraint Bridging and Predictive Inference Texts by Visual Field-Hemisphere

\begin{tabular}{llllll}
\hline & \multicolumn{2}{c}{ rvf-LH } & & \multicolumn{2}{c}{ lvf-RH } \\
\cline { 2 - 3 } \cline { 5 - 6 } Condition & $M$ & $S E$ & & $M$ & $S E$ \\
\hline Bridging Inference Texts & & & & \\
$\quad$ Strong textual constraint & 98 & 0.01 & & 98 & 0.01 \\
Weak textual constraint & 98 & 0.01 & & 98 & 0.01 \\
$\quad$ Neutral textual constraint & 97 & 0.01 & & 92 & 0.01 \\
Predictive Inference Texts & & & & \\
$\quad$ Strong textual constraint & 97 & 0.01 & & 98 & 0.01 \\
Weak textual constraint & 98 & 0.01 & & 96 & 0.01 \\
Neutral textual constraint & 94 & 0.01 & & 91 & 0.02 \\
\hline
\end{tabular}

Note-rvf-LH, right visual field-left hemisphere; lvf-RH, left visual field-right hemisphere.

Follow-up analyses showed that for low working memory capacity readers (see Figure 2, left panel), there was a main effect of textual constraint $\left[F(1,25)=18.18, M S_{\mathrm{e}}=8,024\right.$, $p<.05]$ and a textual constraint $\times$ visual field-hemisphere interaction $\left[F(1,25)=4.75, M S_{\mathrm{e}}=6,620, p<.05\right]$. There was a significant effect of textual constraint only for the rvf-LH $\left[F(1,25)=18.86, M S_{\mathrm{e}}=8,292, p<.05\right]$. The rvf-LH showed greater inference-related facilitation for strongly constrained inferences $(M=74.58, S E=23.33)$ than for weakly constrained inferences $(M=-35.10$, $S E=24.92)$. There was no effect of textual constraint for the lvf-RH $(F<1)$. In addition, weakly constrained inferences showed greater inference-related facilitation in the lvf-RH than in the rvf-LH $\left[F(1,25)=4.26, M S_{\mathrm{e}}=\right.$ $20,179, p<.05]$. There was no difference in inferencerelated facilitation for strongly constrained inferences between the lvf-RH and the rvf-LH $(F<1)$.

For high working memory capacity readers (see Figure 2, right panel), there was a main effect of textual constraint $\left[F(1,26)=14.47, M S_{\mathrm{e}}=10,763, p<.05\right]$. There was greater inference-related facilitation for strongly constrained inferences $(M=77.52, S E=18.13)$ than for weakly constrained ones $(M=1.58, S E=18.38)$ in both the left and the right hemispheres. In addition, there was no difference in inference-related facilitation for weakly constrained inferences in the rvf-LH and lvf-RH $(F<1)$. Likewise, there was no difference in inference-related facilitation for strongly constrained inferences in the lvf- $\mathrm{RH}$ and the $\operatorname{rvf}-\mathrm{LH}(F<1)$.

\section{Working Memory Capacity Effects on Accuracy Response Time Facilitation}

The inference-related facilitation data for accuracy were submitted to a $2 \times 2 \times 2$ (working memory capacity $\times$ textual constraint $\times$ visual field-hemisphere) ANOVA. The overall analysis showed a main effect of visual fieldhemisphere $\left[F(1,51)=16.19, M S_{\mathrm{e}}=0.008, p<.05\right]$. Both hemispheres showed high levels of accuracy facilitation, but the right hemisphere showed more accurate facilitation overall $(M=0.02, S E=0.01)$ than did the left hemisphere $(M=0.06, S E=0.01)$. There were no effects due to individual differences in working memory capacity $(F \mathbf{s}<1)$.

\section{Discussion}

The pattern of results in Experiment 1 indicates that both hemispheres are involved in the generation of bridging inferences when the text provides strong causal constraints and that the right hemisphere is more involved than the left hemisphere in the generation of weakly constrained inferences. Thus, the right hemisphere is activated during the generation of bridging inferences and may even have a special role to play in inference generation when the text provides weak constraints. In addition, Experiment 1 showed that low and high working memory capacity readers differ in terms of right-hemispheric facilitation patterns for strongly and weakly constrained bridging inferences. Specifically, high working memory capacity readers showed evidence of greater inference facilitation for strongly constrained inferences than for weakly constrained inferences in both hemispheres. In contrast, low working memory capacity readers showed this same pattern in the left hemisphere but equal facilitation in the right hemisphere.

These findings were obtained with bridging inferences, which are crucial to building coherence during reading. In Experiment 2, we extended our investigation to a second important type of inferences during reading: those that are predictive of upcoming text. The aim was to investigate whether the observed differences in hemispheric

Table 4

Mean Response Times (in Milliseconds) and Standard Errors for Strong, Weak, and Neutral Textual Constraint Bridging and Predictive Inference Texts by Visual Field-Hemisphere and Working Memory Capacity

\begin{tabular}{|c|c|c|c|c|}
\hline \multirow[b]{3}{*}{ Condition } & \multicolumn{4}{|c|}{ Working Memory Capacity } \\
\hline & \multicolumn{2}{|c|}{ Low } & \multicolumn{2}{|c|}{ High } \\
\hline & $M$ & $S E$ & $M$ & $S E$ \\
\hline
\end{tabular}

Bridging Inference Texts

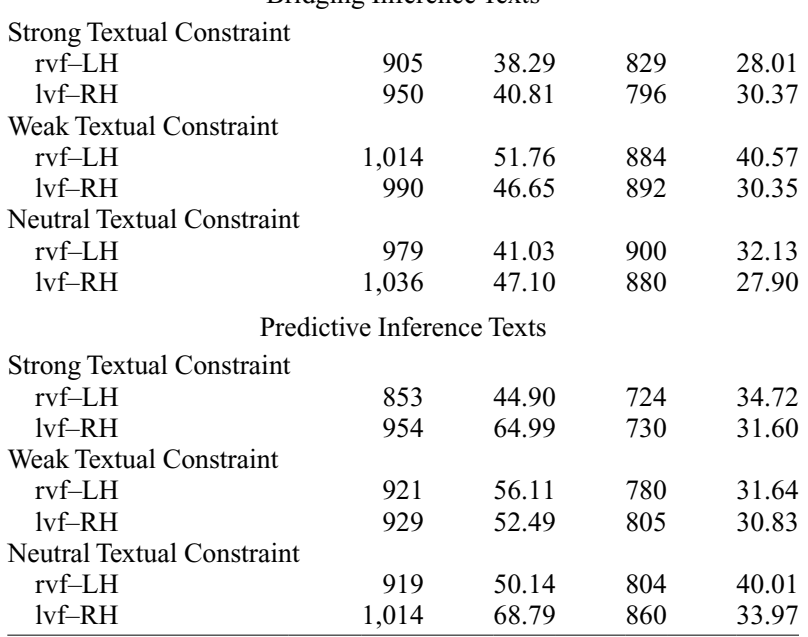

Note-rvf-LH, right visual field-left hemisphere; lvf-RH, left visual field-right hemisphere. 


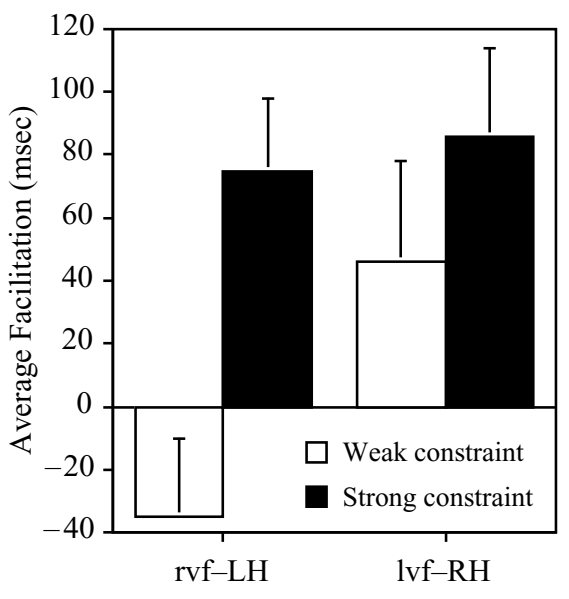

Visual Field-Hemisphere

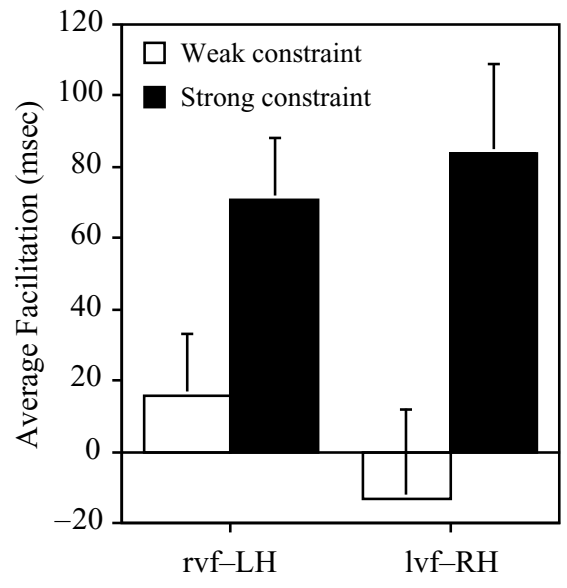

Visual Field-Hemisphere

Figure 2. Average facilitation for strongly and weakly constrained bridging inference texts by visual field-hemisphere and working memory capacity (Experiment 1). The left panel shows facilitation for low working memory capacity readers, and the right panel shows facilitation for high working memory capacity readers. rvf-LH, right visual field-left hemisphere; Ivf-RH, left visual field-right hemisphere.

roles would be evident in the generation of other types of inferences as well. Hemispheric differentiation might be even stronger for predictive inferences than for bridging inferences. For example, prior research has suggested that the right hemisphere produces greater activation for concepts related to predictive inferences than for concepts related to bridging inferences (Beeman et al., 2000). In addition, the processing of predictive inferences appears especially sensitive to the causal constraints provided by a text (Linderholm, 2002). This sensitivity may be due to the fact that predictive inferences are less constrained by a text, whereas bridging inferences tend to be more heavily constrained by a text (van den Broek, 1994; van den Broek, Fletcher, \& Risden, 1993; Vonk \& Noordman, 1990). Thus, hemispheric differentiation as a function of the causal constraints in a text may be particularly salient in the processing of predictive inferences. Furthermore, it was important to explore whether the findings with respect to working memory capacity differences in the processing of bridging inferences would generalize to predictive inferences. These were the aims of Experiment 2.

\section{EXPERIMENT 2}

In Experiment 2, we investigated the influence of the level of textual constraint on the hemispheric processing of predictive inferences. If the level of textual constraint influences the processing of predictive inferences in the hemispheres, the rvf-LH will show high levels of inference-related facilitation for strongly constrained predictive inferences, whereas it will show low levels of inference-related facilitation for weakly constrained predictive inferences. More importantly, we hypothesized that the lvf-RH would show high levels of inference-related facilitation effects for strongly and weakly constrained predictive inferences. Again, we considered whether the effects of textual constraint on hemispheric processing of inferences vary across readers with different working memory capacities.

\section{Method}

\section{Participants}

Seventy-two undergraduate students at the University of Minnesota participated in the experiment in exchange for course credit in an introductory psychology course. Of these, 36 were male, and 36 were female (17-31 years of age). All the participants had normal or corrected-to-normal vision and were native speakers of American English. All were right-handed, as assessed through the Edinburgh Handedness Inventory (mean laterality quotient $=.77$; Oldfield, 1971).

\section{Materials and Procedure}

Experiment 2 followed the same design as that in Experiment 1, except that the 48 inference items promoted predictive inferences and the target words were associated with predictive inferences. Examples of an inference text in its strong and weak constraint versions and of a neutral text are given in Table 5. Again, we presented the texts in a pilot study to 22 participants to verify the causal constraint manipulation. The results confirmed that the target word was more often mentioned as part of the explanations for the strong constraint version $(M=24.33, S E=1.21)$ than for the weak constraint version $(M=8.00, S E=1.07)[t(47)=12.43, S E=1.31, p<.01]$. Importantly, the average responses to the strong and weak constraint texts were significantly greater than would be expected if the readers had been generating inferences by chance. In addition, the average responses to the strong and weak constraint texts were significantly greater than zero $[t(47)=20.10, S E=1.21, p<.05$, and $t(47)=$ $7.50, S E=1.07, p<.05$, respectively $]$.

\section{Reading Span Task}

The participants were administered the same reading span task as that in Experiment 1 (Singer \& Ritchot, 1996). When the upper and lower terciles of the distribution of final words recalled were used, 26 participants were identified as low working memory capacity 
Table 5

Example Predictive Inference Text Used in Experiment 2

Inference Text

Tom and Krista were standing together holding hands.

Both of them were a little nervous, but mostly excited about today.

Tom imagined the future as he looked at Krista.

Strong textual constraint: They were just pronounced as man and wife. Weak textual constraint: They were just announced as college graduates. Target word: kiss

\section{Neutral Text}

The three women had been friends since childhood.

No matter where they were, they stayed in touch.

Currently, they were together to celebrate New Year's Eve.

They spent the evening discussing old memories and talking about the future.

Target word: kiss

readers $(M=28.48, S D=2.93)$, and 27 participants as high working memory capacity readers $(M=39.04, S D=2.20)$.

\section{Results}

\section{Inference-Related Response Time Facilitation Effects}

To test the main hypothesis that the level of textual constraint influences the processing of predictive inferences, we conducted analyses on the inference-related facilitation effects. We obtained the inference-related facilitation effects by calculating the difference between the neutral and the inference condition response times in each visual field-hemisphere. A repeated measures ANOVA was conducted on mean inference-related facilitation and accuracy facilitation. The independent variables were visual fieldhemisphere (left or right) and textual constraint (strong or weak). Sex, response hand, counterbalancing condition, and participant were entered as between-subjects variables for the by-participants analyses (see Pollatsek \& Well, 1995). There was no effect of sex, response hand, or counterbalancing condition, so these analyses will not be reported.

The mean inference-related facilitation for strongly and weakly constrained texts by visual field-hemisphere is presented in Figure 3 (see Table 2 for mean response times in each condition). ${ }^{2}$ There was no main effect of visual field-hemisphere $\left[F_{1}(1,64)=1.40, M S_{\mathrm{e}}=38,380.24\right.$, $\left.p=.24 ; F_{2}(1,47)=0.77, M S_{\mathrm{e}}=33,371.21, p=.38\right]$. There was a main effect of textual constraint $\left[F_{1}(1,64)=\right.$ $16.62, M S_{\mathrm{e}}=121,348.72, p<.05 ; F_{2}(1,47)=5.31$, $\left.M S_{\mathrm{e}}=66,383.27, p<.05\right]$. There was greater inferencerelated facilitation for strongly constrained texts than for weakly constrained texts $(M=93.25, S E=13.02$, and $M=50.22, S E=10.73$, respectively). Most importantly, there was a significant visual field-hemisphere $X$ textual constraint interaction by participants $\left[F_{1}(1,64)=\right.$ $5.06, M S_{\mathrm{e}}=32,346.96, p<.05 ; F_{2}(1,47)=1.45, M S_{\mathrm{e}}=$ 23,261.94, $p=.23$ ]. Simple-effect contrasts revealed that in the rvf-LH, strongly constrained texts showed greater inference-related facilitation than did weakly constrained texts $\left[F_{1}(1,64)=21.81, M S_{\mathrm{e}}=139,499.75\right.$, $\left.p<.05 ; F_{2}(1,47)=5.23, M S_{\mathrm{e}}=84,118.97, p<.05\right]$. In the lvf-RH, there was no difference between inferencerelated facilitation for strongly and weakly constrained texts $\left[F_{1}(1,64)=2.22, M S_{\mathrm{e}}=14,195.93, p=.14\right.$; $\left.F_{2}(1,47)=0.34, M S_{\mathrm{e}}=5,526.25, p=.56\right]$. However, simple-effect contrasts revealed that weakly constrained inferences showed greater inference-related facilitation in the lvf-RH than in the rvf-LH $\left[F_{1}(1,64)=11.04, M S_{\mathrm{e}}=\right.$ $70,598.30, p<.05 ; F_{2}(1,47)=9.29, M S_{\mathrm{e}}=56,178.36$, $p<.05]$. Thus, both hemispheres showed facilitation of predictive inferences when the text provided strong causal constraints, but the right hemisphere showed greater facilitation of predictive inferences than did the left hemisphere when the text provided weak causal constraints.

One-tailed paired difference $t$ tests were conducted on the predictive inference-related facilitation effects to determine whether there was facilitation above zero for each of the inference conditions in each visual field. In the rvf$\mathrm{LH}$, the inference-related facilitation was significantly greater than zero for strongly and weakly constrained texts $\left[t_{1}(71)=6.59, S E=13.89, p<.05\right.$, and $t_{2}(47)=4.38$, $S E=21.79, p<.05 ; t_{1}(71)=2.08, S E=13.61, p<.05$, and $\left.t_{2}(47)=1.94, S E=18.72, p=.06\right]$. In the lvf-RH, the inference-related facilitation was significantly greater than zero for strongly and weakly constrained texts [for strongly constrained texts, $t_{1}(71)=4.30, S E=22.13, p<$ .05 , and $t_{2}(47)=3.56, S E=28.06, p<.05$; for weakly constrained texts, $t_{1}(71)=4.43, S E=16.27, p<.05$, and $\left.t_{2}(47)=3.75, S E=22.57, p<.05\right]$.

Comparison of inference-related response time facilitation effects for bridging inferences (Experiment 1) and predictive inferences (Experiment 2). We compared inference-related facilitation results across

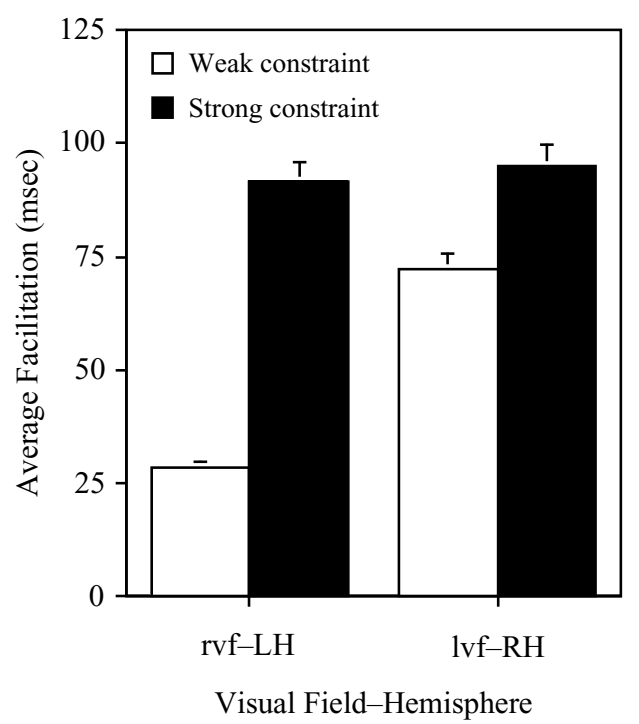

Figure 3. Average facilitation for strongly and weakly constrained predictive inference texts by visual field-hemisphere (Experiment 2). rvf-LH, right visual field-left hemisphere; lvfRH, left visual field-right hemisphere. 
Experiments 1 and 2 in a factorial ANOVA analysis to determine whether hemispheric facilitation differed for bridging and predictive inferences. ${ }^{3}$ The main effect of inference type was marginally significant $[F(1,142)=3.19$, $\left.M S_{\mathrm{e}}=101,442.95, p=.08\right]$. Overall, predictive inferences showed marginally greater inference-related facilitation than did bridging inferences $(M=71.74, S E=8.51$, and $M=45.20, S E=7.55$, respectively). The main effect of visual field-hemisphere was significant $[F(1,142)=$ 5.02, $\left.M S_{\mathrm{e}}=114,614.29, p<.05\right]$. Inference-related facilitation was greater in the lvf-RH than in the rvf-LH $(M=72.57, S E=8.88$, and $M=44.36, S E=7.11$, respectively). The main effect of textual constraint was significant $\left[F(1,142)=62.59, M S_{\mathrm{e}}=525,295.35, p<\right.$ $.05]$. Inference-related facilitation was greater for strongly constrained inferences than for weakly constrained inferences $(M=88.67, S E=8.16$, and $M=28.27, S E=7.60$, respectively). Of primary interest, there was no three-way interaction for visual field-hemisphere, textual constraint, and inference type $\left[F(1,142)=0.87, M S_{\mathrm{e}}=6,337.64\right.$, $p=.35]$. Thus, hemispheric facilitation for strongly and weakly constrained inferences did not differ for bridging and predictive inferences. The only interaction that was significant was the textual constraint $X$ inference type interaction $\left[F(1,142)=5.17, M S_{\mathrm{e}}=43,421.38, p<.05\right]$. However, the latter finding should be interpreted with caution, because the inference texts and target words differed across Experiments 1 and 2. In summary, although predictive inferences showed greater inference-related facilitation than did bridging inferences, there was no evidence of differences between bridging and predictive inferences in terms of hemispheric inference-related facilitation patterns.

\section{Accuracy Response Time Facilitation Effects}

Facilitation of the accuracy responses was inspected to validate the speed-based results (see Table 3 for mean accuracy response times in each inference condition). The main effect of visual field-hemisphere was marginally significant $\left[F(1,64)=2.67, M S_{\mathrm{e}}=0.04, p=.10\right.$; $\left.F_{2}(1,47)=1.55, M S_{\mathrm{e}}=0.02, p=.22\right]$. Overall, accuracy facilitation was marginally greater when target words were presented to the lvf- $\mathrm{RH}$ than when target words were presented to the rvf-LH $(M=6.1 \%, S E=0.01$, and $M=$ $3.7 \%, S E=0.01)$. There was no main effect of textual constraint $\left[F(1,64)=2.14, M S_{\mathrm{e}}=0.01, p=.15 ; F_{2}(1,47)=\right.$ $\left.2.14, M S_{\mathrm{e}}=0.01, p=.15\right]$. The visual field-hemisphere $\times$ textual constraint interaction was marginally significant $\left[F_{1}(1,64)=3.26, M S_{\mathrm{e}}=0.01, p=.08 ; F_{2}(1,47)=3.23\right.$, $\left.M S_{\mathrm{e}}=0.01, p=.08\right]$. It should be noted that accuracy was very high and close to ceiling in the rvf-LH. Thus, there was no evidence of a speed-accuracy trade-off.

\section{Working Memory Capacity Effects on Inference-Related Response Time Facilitation}

To explore the effects of working memory capacity, the predictive-inference-related facilitation data were submitted to a $2 \times 2 \times 2$ (working memory capacity $\times$ textual constraint $\times$ visual field-hemisphere) mixed factors ANOVA (see Table 4 for mean response times in each inference condition). Given that working memory analyses were secondary and involved only a subset of the data, items were not balanced across conditions or participants, and therefore, no by-items analyses will be reported. There was a significant three-way interaction of working memory capacity, textual constraint, and visual field-hemisphere $\left[F(1,49)=5.87, M S_{\mathrm{e}}=6,828, p<.05\right]$.

Follow-up analyses showed that for low working memory capacity readers (see Figure 4, left panel), there was a significant textual constraint $\times$ visual field-hemisphere interaction $\left[F(1,24)=5.81, M S_{\mathrm{e}}=9,416, p<.05\right]$. The main effect of textual constraint was significant only for the rvf-LH $\left[F(1,24)=7.11, M S_{\mathrm{e}}=8,289, p<.05\right]$. Low working memory capacity readers showed greater rvf$\mathrm{LH}$ facilitation for strongly constrained inferences $(M=$ $66.51, S E=18.48$ ) than for weakly constrained inferences $(M=-2.14, S E=21.93)$. There was no effect of textual constraint for the lvf-RH $(F<1)$. In addition, weakly constrained inferences showed greater inference-related facilitation in the lvf-RH than in the rvf-LH $[F(1,24)=$ 5.32, $\left.M S_{\mathrm{e}}=17,859, p=.03\right]$. There was no difference in inference-related facilitation for strongly constrained inferences between the lvf-RH and the rvf-LH $(F<1)$.

For high working memory capacity readers (see Figure 4, right panel), the only significant finding was a main effect of textual constraint $\left[F(1,25)=18.54, M S_{\mathrm{e}}=\right.$ $6,092, p<.001]$. High working memory capacity readers showed greater facilitation for strongly constrained inferences $(M=105.03, S E=18.22)$ than for weakly constrained inferences $(M=39.11, S E=12.87)$ in both the left and the right hemispheres. In addition, there was no difference in inference-related facilitation for weakly or strongly constrained inferences in the rvf-LH and the lvf-RH, respectively $\left(F_{\mathrm{S}}<1\right)$.

\section{Working Memory Capacity Effects on Accuracy Response Time Facilitation}

The accuracy inference-related facilitation data were submitted to a $2 \times 2 \times 2$ (working memory capacity $\times$ textual constraint $\times$ visual field-hemisphere) ANOVA. The overall analysis showed no effects due to textual constraint, hemispheric processing, or individual differences in working memory capacity $\left(F_{\mathrm{S}}<1\right)$.

\section{Discussion}

The pattern of results in Experiment 2 indicates that both hemispheres are involved in the generation of predictive inferences when the text provides strong causal constraints and that the right hemisphere is more involved than the left hemisphere in the generation of weakly constrained inferences. Therefore, textual constraint differentially influences the hemispheric processing of predictive inferences. In addition, Experiment 2 showed that low and high working memory capacity readers differ in terms of right-hemispheric facilitation patterns for weakly constrained predictive inferences. Specifically, 


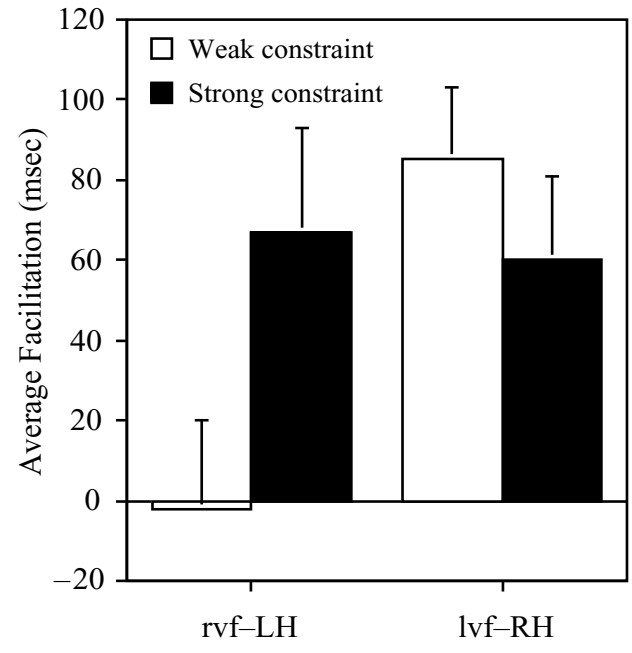

Visual Field-Hemisphere

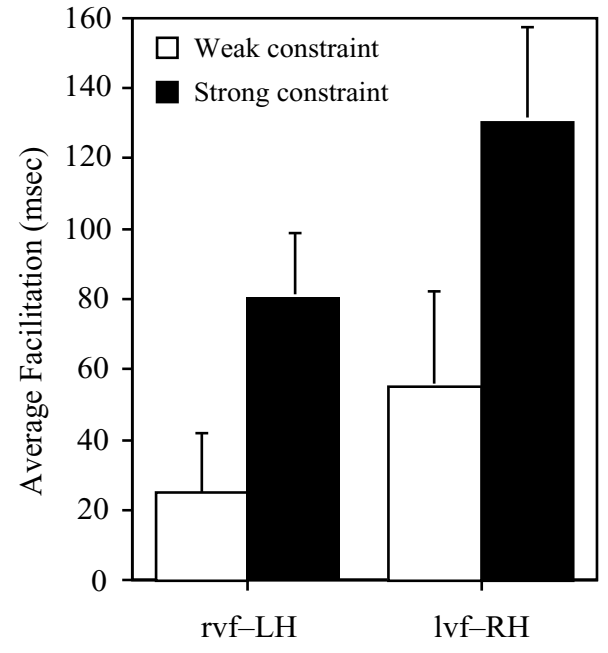

Visual Field-Hemisphere

Figure 4. Average facilitation for strongly and weakly constrained predictive inference texts by visual field-hemisphere and working memory capacity (Experiment 2). The left panel shows facilitation for low working memory capacity readers, and the right panel shows facilitation for high working memory capacity readers. rvf-LH, right visual field-left hemisphere; lvf- $\mathrm{RH}$, left visual field-right hemisphere.

high working memory capacity readers showed evidence of greater inference facilitation for strongly constrained inferences than for weakly constrained inferences in both hemispheres. In contrast, low working memory capacity readers showed this same pattern in the left hemisphere but equal facilitation in the right hemisphere. The pattern of results for predictive inferences closely matches that obtained for bridging inferences.

\section{GENERAL DISCUSSION}

The results of this study demonstrate that both hemispheres play a role in the processing of bridging and predictive inferences. For strong causally constrained inferences, both hemispheres showed high levels of inference-related facilitation in a lexical decision task. In contrast, for weak causally constrained inferences, the right hemisphere showed stronger facilitation than did the left hemisphere.

The results-in particular, those of Experiment 2, which showed the clearest differentiation in activation patterns between the left and the right hemispheres - suggest that two separable mechanisms, located in different hemispheres, may be involved in the generation of weakly and strongly constrained predictive inferences. These results argue against the possibility that the processing of particular types of inferences takes place in one location of the brain, with variations in constraint simply affecting the speed of processing or the amount of activation involved. If this had been the case, we should have observed the same patterns of hemispheric facilitation for a particular inference type regardless of the causal constraint provided by the text, although the absolute level of activation might have changed.
The viewpoint that different levels of textual constraint represent qualitatively different processes is consistent with other recent findings. For example, the level of syntactic and semantic constraint in the hemispheres has been examined. Consistent with our results, the left hemisphere is more sensitive to sentences ending with highly expected words, sentences with congruent endings, and well-structured sentences than is the right hemisphere (Faust et al., 1995; Faust \& Kravetz, 1998). Therefore, the left hemisphere may be heavily involved in the processing of semantic information in highly constrained contexts, whereas the right hemisphere may be heavily involved in semantic processing in both less and more constrained contexts. The evidence from the present and other investigations points to the possibility that two different mechanisms exist and that these mechanisms operate to different degrees in the left and the right hemispheres of the brain.

The notion that the right hemisphere plays a unique role in the generation of weakly constrained inferences is consistent with the fine-coarse semantic coding theory (Beeman et al., 1994). The results in the present study suggest that textual constraint can be added to this theory by incorporating the strength of the connection between semantic concepts that results from the causal constraint present in a text. That is, our data suggest that the connection strength between semantic concepts varies as a function of textual constraint. According to this explanation, the left hemisphere creates less overlap but stronger connections between semantic concepts, resulting in faster processing of a limited number of highly constrained inferences. In contrast, the right hemisphere creates greater overlap but weaker connections between semantic concepts, resulting in slower processing of a larger number of weakly constrained inferences. Thus, textual constraint 
determines the degree of semantic overlap, which, in turn, determines the degree of processing in the left and right hemispheres.

Our findings also suggest that hemispheric activation depends not on the type of inference (i.e., bridging or predictive) per se, but on whether the inference is strongly or weakly constrained by the text. Thus, insofar as predictive inferences tend to be less constrained by information in the text than are bridging inferences, one would expect inference-related facilitation patterns to be more robust for predictive inferences than for bridging inferences. This interpretation of our findings can also account for previous investigations showing greater activation in the left hemisphere for bridging inferences and greater activation in the right hemisphere for predictive inferences (Beeman et al., 2000). The generally more constrained nature of bridging inferences may tend to lead to greater left-hemisphere activation, whereas the generally less constrained nature of predictive inferences may tend to lead to greater righthemisphere activation.

Although the interaction between textual constraint and hemispheric processing was the main focus of this article, we also considered potential connections to individual differences, particularly those due to working memory capacity. This was prompted by the finding that low and high working memory capacity readers differentially process inferences that are weakly and strongly constrained by a text (Linderholm, 2002). In the present study, we observed differences between low and high working memory capacity readers in the right hemisphere, but not in the left. With respect to the right hemisphere, low working memory capacity readers showed equally strong facilitation for weakly and strongly constrained inferences, whereas high working memory capacity readers showed more facilitation for strongly constrained inferences than for weakly constrained inferences. With respect to the left hemisphere, both low and high working memory capacity readers showed more facilitation for strongly constrained inferences than for weakly constrained inferences. These findings raise the possibility that high working memory capacity readers may activate fewer weakly constrained inferences in the right hemisphere than do other readers. Indeed, one could speculate that readers have high working memory capacities precisely because they activate less information that may not be as crucial for comprehension, as is the case with weakly constrained inferences. This notion would be consistent with theories of individual differences in working memory capacity that claim that higher working memory capacity readers may be more efficient at allocating attentional resources to the most task-relevant information (Engle, 2002). Thus, the present study extends previous findings by suggesting that a reader's working memory capacity affects how textual constraint is processed in the hemispheres.

In illuminating the role of textual constraint and working memory capacity in the hemispheric processing of inferences, this investigation adds to our knowledge of text comprehension. As individuals read a text, each hemi- sphere may be specialized for processing specific types of semantic information. The left hemisphere appears to be highly activated in strongly constrained conditions, whereas the right hemisphere appears to be activated in both weakly and strongly constrained conditions. The activation of the left hemisphere only in strongly constrained conditions suggests that this hemisphere may be important for information that is explicitly stated in a text, and the activation of the right hemisphere under both weakly and strongly constrained conditions suggests that the right hemisphere may be important for processing information that is not necessarily explicitly mentioned in a text. This conceptualization is consistent with text comprehension theories proposing that readers construct two different representations of the meaning of a text: a text base representation, which mostly contains explicit text, and a situation model representation, which also includes the reader's semantic knowledge (Graesser et al., 1994; Kintsch \& van Dijk, 1978; McKoon \& Ratcliff, 1989). Recently, it has been proposed that text base representations are derived primarily from the left hemisphere, whereas situation model representations are derived from both hemispheres (Long \& Baynes, 2002). Consistent with this viewpoint, our results suggest that both hemispheres activate semantic concepts necessary for the creation of a situation model representation. In addition, the mediating factors of textual constraint and working memory capacity influence how linguistic information is processed in the hemispheres during causal inference generation. Thus, the present findings contribute toward a neurological model of text comprehension, indicating that the right hemisphere is involved in bridging and predictive inference generation in a unique way. Furthermore, the right hemisphere may be a source of individual differences in readers and may play a critical role in activating both strong and weak causally constrained information, which is crucial in the formation of a situation model representation during text comprehension.

\section{REFERENCES}

BeEman, M. [J.] (1993). Semantic processing in the right hemisphere may contribute to drawing inferences during comprehension. Brain \& Language, 44, 80-120.

Beeman, M. J., Bowden, E. M., \& Gernsbacher, M. A. (2000). Right and left hemisphere cooperation for drawing predictive and coherence inferences during normal story comprehension. Brain \& Language, 71, 310-336.

Beeman, M. [J.], Friedman, R. B., Grafman, J., Perez, E., Diamond, S., \& LindSAY, M. B. (1994). Summation priming and coarse semantic coding in the right hemisphere. Journal of Cognitive Neuroscience, 6, 26-45.

Bottini, G., Corcoran, R., Sterzi, R., Paulesu, E., Schenone, P., SCARPA, P., ET AL. (1994). The role of the right hemisphere in the interpretation of figurative aspects of language: A positron emission tomography activation study. Brain, 117, 1241-1253.

Brownell, H. H., Potter, H. H., Bihrle, A. M., \& Gardner, H. (1986). Inference deficits in right brain-damaged patients. Brain \& Language, 27, 310-321.

Chiarello, C., Senehi, J., \& Soulier, M. (1986). Viewing conditions and hemisphere asymmetry for the lexical decision. Neuropsychologia, 24, 521-529. 
Church, K. L., \& Chiarello, C. (1988). Lateral eyes or lateralized? Hemiretinal and ocular dominance effects on visual field asymmetries for the lexical decision task. Brain \& Cognition, 8, 227-239.

Coney, J. (2002). Probing hemispheric processes in an on-line reading task. Brain \& Language, 80, 130-141.

Cook, A. E., Limber, J. E., \& O'Brien, E. J. (2001). Situation-based context and the availability of predictive inferences. Journal of Memory \& Language, 44, 220-234.

ENGLE, R. W. (2002). Working memory capacity as executive attention. Current Directions in Psychological Science, 11, 19-23.

Faust, M., BabKoff, H., \& Kravetz, S. (1995). Linguistic processes in the two cerebral hemispheres: Implications for modularity vs interactionism. Journal of Clinical \& Experimental Neuropsychology, 17, 171-192.

Faust, M., \& Kravetz, S. (1998). Levels of sentence constraint and lexical decision in the two hemispheres. Brain \& Language, 62, 149-162.

Faust, M., Kravetz, S., \& BabKoff, H. (1993). Hemisphericity and top-down processing of language. Brain \& Language, 44, 1-18.

FINCHER-KIEFER, R. (1995). Relative inhibition following the encoding of bridging and predictive inferences. Journal of Experimental Psychology: Learning, Memory, \& Cognition, 21, 981-995.

Francis, W. N., \& KučERA, H. (1982). Frequency analysis of English usage: Lexicon and grammar. Boston: Houghton Mifflin.

Graesser, A. C., \& Clark, L. F. (1985). Structures and procedures of implicit knowledge. Norwood, NJ: Ablex.

Graesser, A. C., Singer, M., \& Trabasso, T. (1994). Constructing inferences during narrative text comprehension. Psychological Review, 101, 371-395.

IACOBONI, M., \& ZAIDEL, E. (1996). Hemispheric independence in word recognition: Evidence from unilateral and bilateral presentations. Brain \& Language, 53, 121-140.

Johnson, M. K., Bransford, J. D., \& Solomon, S. K. (1973). Memory for tacit implications of sentences. Journal of Experimental Psychology, 98, 203-205.

KINTSCH, W., \& VAN DIJK, T. A. (1978). Toward a model of text comprehension and production. Psychological Review, 85, 363-394.

Linderholm, T. (2002). Predictive inference generation as a function of working memory capacity and causal text constraints. Discourse Processes, 34, 259-280.

Linderholm, T., \& VAN DEN BRoeK, P. (2002). The effects of reading purpose and working memory capacity on the processing of expository text. Journal of Educational Psychology, 94, 778-784.

LONG, D. L., \& BAYNES, K. (2002). Discourse representation in the two cerebral hemispheres. Journal of Cognitive Neuroscience, 14, 228-242.

McKoon, G., \& Ratcliff, R. (1989). Semantic associations and elaborative inference. Journal of Experimental Psychology: Learning, Memory, \& Cognition, 15, 326-338.

McKoon, G., \& Ratcliff, R. (1992). Inference during reading. Psychological Review, 99, 440-466.

Monzó, A. E., \& Calvo, M. G. (2002). Context constraints, prior vocabulary knowledge and on-line inferences in reading. Psicothema, 14, 357-362.

Myers, J. L., Shinjo, M., \& Duffy, S. A. (1987). Degree of causal relatedness and memory. Journal of Memory \& Language, 26, 453-465.

O’Brien, E. J., Shank, D. M., Myers, J. L., \& Rayner, K. (1988). Elaborative inferences during reading: Do they occur on-line? Journal of Experimental Psychology: Learning, Memory, \& Cognition, 14, $410-420$.

OLDFIELD, R. C. (1971). The assessment and analysis of handedness: The Edinburgh inventory. Neuropsychologia, 9, 97-113.

Pollatsek, A., \& Well, A. D. (1995). On the use of counterbalanced designs in cognitive research: A suggestion for a better and more powerful analysis. Journal of Experimental Psychology: Learning, Memory, \& Cognition, 21, 785-794.

Robertson, D. A., Gernsbacher, M. A., Guidotti, S. J., Robertson, R. R., Irwin, W., Mock, B. J., \& Campana, M. E. (2000). Functional neuroanatomy of the cognitive process of mapping during discourse comprehension. Psychological Science, 11, 255-260.
Singer, M. (1990). Psychology of language: An introduction to sentence and discourse processes. Hillsdale, NJ: Erlbaum.

Singer, M., Andrusiak, P., Reisdorf, P., \& Black, N. L. (1992). Individual differences in bridging inference processes. Memory \& Cognition, 20, 539-548.

Singer, M., \& Ferreira, F. (1983). Inferring consequences in story comprehension. Journal of Verbal Learning \& Verbal Behavior, 22, 437-448.

SingER, M., \& Ritchot, K. F. M. (1996). The role of working memory capacity and knowledge access in text inference processing. Memory \& Cognition, 24, 733-743.

St. George, M., Kutas, M., Martinez, A., \& Sereno, M. I. (1999). Semantic integration in reading: Engagement of the right hemisphere during discourse processing. Brain, 122, 1317-1325.

St. George, M., Mannes, S., \& Hoffman, J. E. (1997). Individual differences in inference generation: An ERP analysis. Journal of Cognitive Neuroscience, 9, 776-787.

Till, R. E., Mross, E. F., \& Kintsch, W. (1988). Time course of priming for associate and inference words in a discourse context. Memory \& Cognition, 16, 283-298.

vAN DEN BROEK, P. (1990). The causal inference maker: Towards a process model of inference generation in text comprehension. In D. A. Balota, G. B. Flores d'Arcais, \& K. Rayner (Eds.), Comprehension processes in reading (pp. 423-445). Hillsdale, NJ: Erlbaum.

VAN DEN BRoEK, P. (1994). Comprehension and memory for narrative texts: Inferences and coherence. In M. A. Gernsbacher (Ed.), Handbook of psycholinguistics (pp. 539-588). San Diego: Academic Press.

VAN DEN Broek, P., Fletcher, C. R., \& Risden, K. (1993). Investigations of inferential processes in reading: A theoretical and methodological integration. Discourse Processes, 16, 169-180.

van DEN Broek, P., \& HuAng, Y.-F. (1995, November). Forward inferences during text comprehension: The role of causal constraints. Paper presented at the 36th Annual Meeting of the Psychonomic Society, Los Angeles.

Vonk, W., \& Noordman, L. G. M. (1990). On the control of inferences in text understanding. In D. A. Balota, G. B. Flores d'Arcais, \& K. Rayner (Eds.), Comprehension processes in reading (pp. 447-464). Hillsdale, NJ: Erlbaum.

\section{NOTES}

1. In general, the left hemisphere has a well-documented advantage over the right hemisphere in language tasks. Indeed, for the neutral condition, in which the task consisted solely of word identification, such an advantage was observed in the present study: The left hemisphere responded more quickly than the right hemisphere for bridging inferences $\left[F(71)=5.10, M S_{\mathrm{e}}=30,912.06, p<.05\right]$. Interestingly, this effect is not as strong for the inference condition. For the weakly constrained bridging inferences, response times did not differ significantly between the left and the right hemispheres $\left[F(71)=0.67, M S_{\mathrm{e}}=4,077.51, p=\right.$ .41]. For the strongly constrained bridging inferences, response times also did not differ between the left and the right hemispheres $[F(71)=$ $\left.0.08, M S_{\mathrm{e}}=488.51, p=.77\right]$. These findings confirm the general observation that the left hemisphere has an advantage in language tasks, unless the tasks involve inference making. When inferences are involved, the right hemisphere seems to be very capable.

2. A pattern of response time results similar to that for bridging inferences was found for predictive inferences. The left hemisphere responded more quickly than the right hemisphere for predictive inferences $\left[F(71)=14.04, M S_{\mathrm{e}}=109,003.44, p<.05\right]$. Again, this effect was not as strong for the inference condition. For the weakly constrained predictive inferences, response times did not differ significantly between the left and the right hemispheres $\left[F(71)=0.54, M S_{\mathrm{e}}=4,154.23, p=\right.$ .47]. For the strongly constrained predictive inferences, response times were significantly faster in the left than in the right hemisphere $[F(71)=$ 13.09, $\left.M S_{\mathrm{e}}=101,635.53, p<.05\right]$.

3 . We thank an anonymous reviewer for this suggestion. 


\section{APPENDIX A \\ Sample Bridging Inference Texts Used in Experiment 1}

\section{Inference Text}

A Canadian tour group stepped off the bus and onto one of the most gorgeous beaches in Florida.

Most of them had never been this far south, and were excited about exploring the beach.

A few of the tourists laid down on their towels on the sunny beach.

Strong textual constraint: After only one hour, they were pink and could barely move.

Weak textual constraint: After only one hour, they were relaxed and could barely move.

Target word: burn

\section{Inference Text}

Tom and his colleague, Tanya, were out to lunch at Sammy Wong's Restaurant.

The waiter promptly served Tanya her lunch.

Her eyes grew wide when she saw a cockroach in her food.

Strong textual constraint: The entire restaurant was surprised by the noise that she made.

Weak textual constraint: The entire restaurant was surprised by the fuss that she made.

Target word: scream

\section{Inference Text}

Walter was playing with his toy airplane next to the living room window.

He liked to pretend that it was a high-speed jet and could fly over 500 miles per hour.

As Walter was playing with his toy airplane, it flew out of the third-floor window.

Strong textual constraint: Walter looked at the pieces of his toy airplane on the street below.

Weak textual constraint: Walter looked at his favorite, toy airplane on the street below.

Target word: break

\section{Inference Text}

Hugo made pizzas for a living, but really aspired to do something more creative.

After work, Hugo went to the park near his apartment.

He saw a beautiful sunset over the lake and took out his pad of white paper.

Strong textual constraint: After several minutes, the beauty of the sunset was expressed in his brushstrokes.

Weak textual constraint: After several minutes, the beauty of the sunset was expressed in his work.

Target word: paint 


\section{APPENDIX B \\ Sample Predictive Inference Texts Used in Experiment 2}

\section{Inference Text}

Tom was late for school.

He had an early class and had trouble getting up in the morning.

He went to the bus stop, hoping that the bus hadn't left yet.

Strong textual constraint: As he arrived at the bus stop, he saw his bus was just pulling away.

Weak textual constraint: As he arrived at the bus stop, he saw his bus was already 5 blocks away.

Target word: run

\section{Inference Text}

Jeff needed to pass this exam in order to get an A in the course.

His instructor passed out the exam to the class.

Jeff's hands were shaking as he read the first question.

Strong textual constraint: Jeff realized that he had a clear view of another student's answers.

Weak textual constraint: Jeff realized that he had not prepared well enough for the exam.

Target word: cheat

\section{Inference Text}

Amy's new car had a stick shift and she felt a bit insecure about driving it.

When she got home from school, she parked the car and went inside the house.

Then she realized that she had forgotten to put the emergency brake on.

Strong textual constraint: As she looked outside, she saw that her car had been parked on a steep hill.

Weak textual constraint: As she looked outside, she saw that her car was parked in the driveway.

Target word: roll

\section{Inference Text}

Brad was looking for a present for his wife's birthday.

He wanted to find something special for her, but he couldn't afford to buy anything nice.

In the accessories department, he saw an expensive scarf sitting on the counter.

Strong textual constraint: Next, he made his way to the counter and took out his bag.

Weak textual constraint: Next, he made his way to the counter and examined it more closely.

Target word: steal

(Manuscript received October 21, 2002;

revision accepted for publication June 28, 2005.) 